\title{
Note sur l'étalonnage de radiothermomètres infrarouges portables
}

\author{
M Verbrugghe, G Guyot
}

INRA Bioclimatologie, STEFCE, BP 91, 84143 Montfavet, France

(Reçu le 27 décembre 1990; accepté le 25 novembre 1991)

\begin{abstract}
Résumé - La température indiquée par un radiothermomètre infrarouge portable est liée linéairement à celle des objets qui sont visés. Mais, selon les conditions dans lesquelles ces appareils sont utilisés, la pente et l'ordonnée à l'origine des droites d'étalonnage peuvent varier de façon importante. Ainsi, il n'est pas possible d'utiliser les résultats d'étalonnage effectués au laboratoire pour des mesures sur le terrain. Ces variations s'expliquent par des défauts de conception des appareils. Aussi, pour pouvoir effectuer des mesures valables sur le terrain, une méthode simple d'étalonnage in situ est proposée.
\end{abstract}

infrarouge thermique / radiothermomètre / étalonnage / télédétection

Summary - Note on calibration of portable infrared radiothermometers. The temperature indicated by a portable radiothermometer is linearly related to the radiative temperature of the object viewed. But the slope and the origin of the calibration line can vary according to the measurement conditions. For this reason it is not possible to use calibrations obtained in the laboratory for field undertaking measurements. The changes in the calibration lines are explained by faulty equipment design. A simple in situ calibration method is therefore proposed for performing field measurements.

thermal infrared / radiothermometer / calibration / remote sensing

\section{INTRODUCTION}

Les radiothermomètres portables sont des instruments destinés à mesurer, à distance, la température de surface d'objets à partir de l'énergie qu'ils émettent dans le domaine de l'infrarouge thermique. Ils sont utilisés en agronomie pour mesurer les températures de surface de végétaux ou de sols; ils sont généralement autonomes et portables et bien adaptés aux études nécessitant un échantillonnage (Gaussorgues, 1984).

La détermination des températures de surface à partir des mesures faites à l'aide de ces radiothermomètres nécessite leur étalonnage. Ce dernier est généralement réalisé en laboratoire à l'aide de corps noirs à température contrôlée et/ou, plus rarement, sur le terrain, au moyen d'une source thermique de référence (corps noir, eau, etc). Dès 1969, Jackson et Idso (1969) puis Robinson et al (1981) et Jackson et Robinson (1985) mettaient en évidence certains effets de la température ambiante sur la mesure des températures de surface par thermométrie infrarouge. En particulier, la détérioration du signal mesuré au-delà de certains seuils de températures internes du radiothermomètre était soulignée. L'étalonnage de certains radiothermomètres est parfois réalisé à partir de la mesure in situ de leur température interne et des corrélations établies au laboratoire pour différentes températures internes, entre le signal mesuré et la température d'un corps noir (Lagouarde, 1979). Cette méthode ne peut être utilisée que si la courbe d'étalonnage est, pour une température interne donnée, indépendante des conditions d'étalonnage (laboratoire ou terrain). Ces conditions d'utilisation sont difficiles à mettre en œuvre sur le terrain, en particulier lors de mesures impliquant un échantillonnage spatial. Une autre méthode consiste à mesurer la température radiative d'un corps noir sur le terrain, au moment des mesures, et à étalonner le radiothermomètre au laboratoire après l'expérimentation à l'aide d'un corps noir de précision (Kustas et al, 1990). Cette méthode ne peut être utilisée que si le signal délivré par le radiother- 
momètre est indépendant des variations des conditions dans lesquelles il est utilisé.

L'objet de cette note est de montrer l'importance des erreurs qui peuvent être commises sur la mesure des températures de surface, dans les conditions naturelles, et ensuite de proposer une méthode qui permette de limiter ces erreurs.

\section{LES RADIOTHERMOMĖTRES UTILISÉS}

Deux radiothermomètres portables type «pistolet», ont été utilisés (AGA Thermopoint 80 et Mikron série 80 ).

\section{Principe de fonctionnement des radiothermomètres}

Les 2 instruments utilisés sont basés sur le même principe de fonctionnement et ont des caractéristiques techniques voisines. Le flux de rayonnement provenant de la source visée est focalisé sur un détecteur précédé par un filtre, destiné à ne laisser passer que le rayonnement infrarouge thermique $(8-14 \mathrm{~m}$ pour le radiothermomètre $A G A^{*}$ et $7-20 \mathrm{~m}$ pour le radiothermomètre Mikron**) (fig 1). Le système de focalisa tion est constitué par un simple miroir parabolique (Mikron) ou un télescope type cassegrain (AGA). Le détecteur utilisé dans les 2 cas est le même : il est constitué par une microthermopile encapsulée dans un boîtier de transistor qui possède, sur le dessus, une fenêtre jouant le rôle de filtre. Ce type de thermopile est très sensible mais son coefficient de réponse varie avec la température (diminution de la sensibilité de l'ordre de $1 \%$ par ${ }^{\circ} \mathrm{C}$ ). En première approximation, le signal délivré par la thermopile dépend de l'écart de température entre sa surface réceptrice (peinte en noir) et celle de l'objet visé. La surface réceptrice est à une température voisine de celle du boîtier dans lequel la thermopile est encapsulée. Pour mesurer la température radiative d'un objet, il faut donc connaître la tension délivrée aux bornes de la thermopile et la température de son boîtier. Par ailleurs, pour assurer une bonne stabilité des mesures, il est préférable d'insérer le détecteur dans un bloc métallique ayant une assez grande inertie thermique.
Les thermistances indiquées sur la figure 1 servent ainsi à mesurer la température de référence. Celle-ci peut d'ailleurs être utilisée également pour corriger la variation de la sensibilité de la thermopile en fonction de la température.

\section{Problèmes posés par l'utilisation des radiothermomètres sur le terrain}

La figure 1 montre tout d'abord que le radiothermomètre AGA est mieux conçu en ce qui concerne la mesure de la température du boîtier de la thermopile et la stabilisation de celle-ci. La fixation du détecteur du radiothermomètre Mikron dans un bloc de résine (isolante) doit entraîner des dérives importantes, liées à son échauffement. De plus ces échauffements seront mal pris en compte car la thermistance n'est pas en contact thermique avec le détecteur. Le problème le plus important, pour des appareils qui sont utilisés sur le terrain, est l'échauffement du filtre, destiné à ne laisser passer que le rayonnement infrarouge thermique et à absorber le rayonnement solaire (Amiro et al, 1973). En effet, lorsque les mesures sont effectuées au champ, le flux de rayonnement d'origine solaire réfléchi par la végétation est du même ordre de grandeur que le flux de rayonnement thermique émis. Il est absorbé par le filtre qui s'échauffe et émet un rayonnement thermique parasite qui
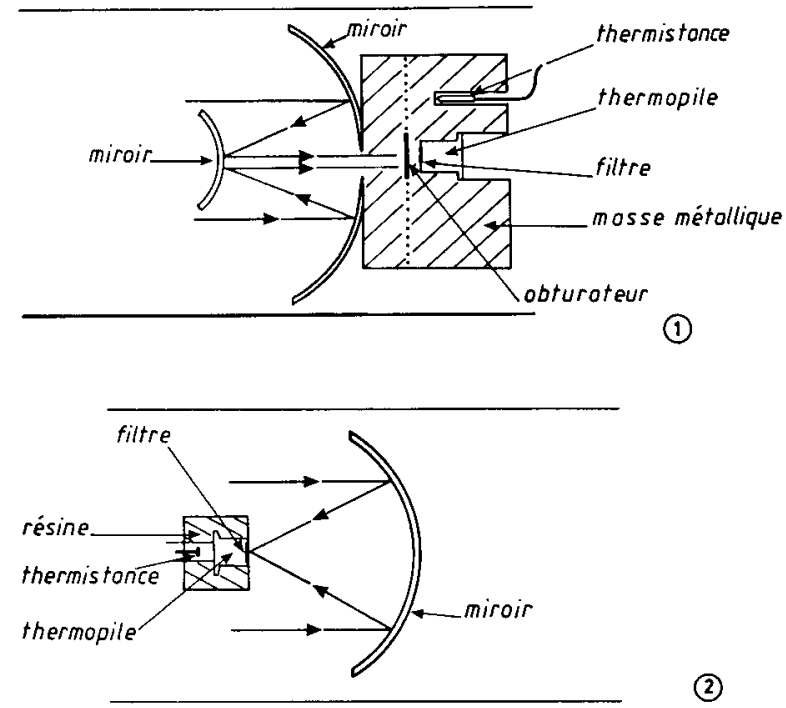

Fig 1. Schéma de principe du système optique des radiothermomètres AGA et MIKRON.

\footnotetext{
" AGA Infrared Systems AB Box 3, S-18211 Dandery Suède.

** Mikron Instrument Co Inc 445 West Main Street Wyckoff, New Jersey 07481
} 
vient se superposer au rayonnement provenant de l'objet visé. On doit donc assister à une augmentation progressive de l'ordonnée à l'origine de la courbe d'étalonnage du radiothermomètre. Ce phénomène sera amplifié si la chaleur ainsi captée par le filtre est difficilement évacuée, ce qui est le cas pour le radiothermomètre Mikron. II faut noter sur la figure 1 que le radiothermomètre AGA est muni d'un obturateur avec une face noire vers le détecteur et une face réfléchissante vers l'extérieur. Il est fermé entre 2 mesures, ce qui limite l'échauffement du filtre de la thermopile.

Les courbes d'étalonnage des radiothermomètres pouvant être assimilées à des droites, nous pouvons donc nous attendre à ce que leur ordonnée à l'origine et leur pente changent en fonction des conditions dans lesquelles ces instruments sont utilisés. C'est ce que nous avons voulu vérifier en comparant des étalonnages effectués au laboratoire (en lumière atténuée) et sur le terrain (autour du midi solaire) où ces appareils sont toujours utilisés en régime transitoire et non en régime permanent (Graham et al, 1989).

\section{COMPARAISON DES ÉTALONNAGES EFFECTUÉS AU LABORATOIRE ET SUR LE TERRAIN}

\section{Mise en œuvre des radiothermomètres}

Les mesures avec les 2 appareils sont pilotées par un microprocesseur qui échantillonne les données durant toute la période pendant laquelle l'opérateur appuie sur le bouton de mise en route. Le radiothermomètre Mikron mémorise la valeur maximale de la température de surface alors que le radiothermomètre AGA indique les valeurs maximale, minimale et moyenne. Ce dernier appareil possède en plus un système de prise en compte de la température de l'environnement lorsque celle-ci est nettement plus élevée que sa température interne (ce dispositif est plus spécialement conçu pour des environnements industriels : fours, machines thermiques, etc). Dans notre cas nous n'avons pas utilisé ce dispositif de correction.

Les 2 radiothermomètres sont munis d'un dispositif qui permet de tenir compte de l'émissivité de la surface visée.

Dans notre cas nous avons pris l'émissivité égale à 0,97 pour la végétation et à 0,98 pour l'eau. Les mesures sur le terrain ayant été effec- tuées au mois de juillet, l'échauffement du corps du radiothermomètre pouvait affecter de façon sensible sa réponse. Aussi, pour limiter cet échauffement, les radiothermomètres ont été enroulés dans 2 feuilles de mousse de polyuréthane $(0,9 \mathrm{~cm})$ recouvertes d'un film de PVC aluminisé. De cette manière, la plus grande partie du rayonnement solaire était réfléchie.

\section{Étalonnage des radiothermomètres}

Nous avons utilisé comme "corps noir» une surface d'eau dont la température était connue avec précision. Pour cela, nous avons employé au laboratoire comme sur le terrain 2 boîtes isothermes cylindriques en PVC de $14 \mathrm{~cm}$ de diamètre et de $17 \mathrm{~cm}$ de hauteur (diamètre de la pupille d'entrée des radiothermomètres : environ $5 \mathrm{~cm}$ ) contenant de l'eau dont on faisait varier la température par mélange d'eau froide et chaude. L'eau était fortement agitée si bien que la température, mesurée à l'aide d'un thermomètre de précision plongé dans celle-ci, était pratiquement égale à celle de la couche superficielle visée par le radiothermomètre.

\section{Résultats des mesures}

Les radiothermomètres ont été étalonnés sur le terrain durant une période de $16 \mathrm{j}$ de beau temps du 9 au 27 juillet 1990 avec cependant 3 journées au cours desquelles un seul des deux appareils a été utilisé. Aussi, les gammes de températures correspondantes ne sont pas tout à fait identiques. De plus, les radiothermomètres ont été étalonnés sur le terrain et au laboratoire les 16 et 23 juillet.

Lorsque les étalonnages sur le terrain ont été effectués, les radiothermomètres avaient été préalablement mis en équilibre thermique avec l'air et avaient été utilisés pour une série de mesures de température de surface de la végétation (pendant environ $30 \mathrm{~min}$ ). Les filtres des détecteurs avaient donc eu la possibilité de s'échauffer.

La figure 2 rassemble les résultats des mesures effectuées sur le terrain. Elle montre que, pour une température donnée de l'eau, on observe une dispersion relativement importante des températures indiquées par les radiothermomètres, avec cependant, une dispersion plus importante pour le radiothermomètre Mikron que pour le radiothermomètre AGA. 

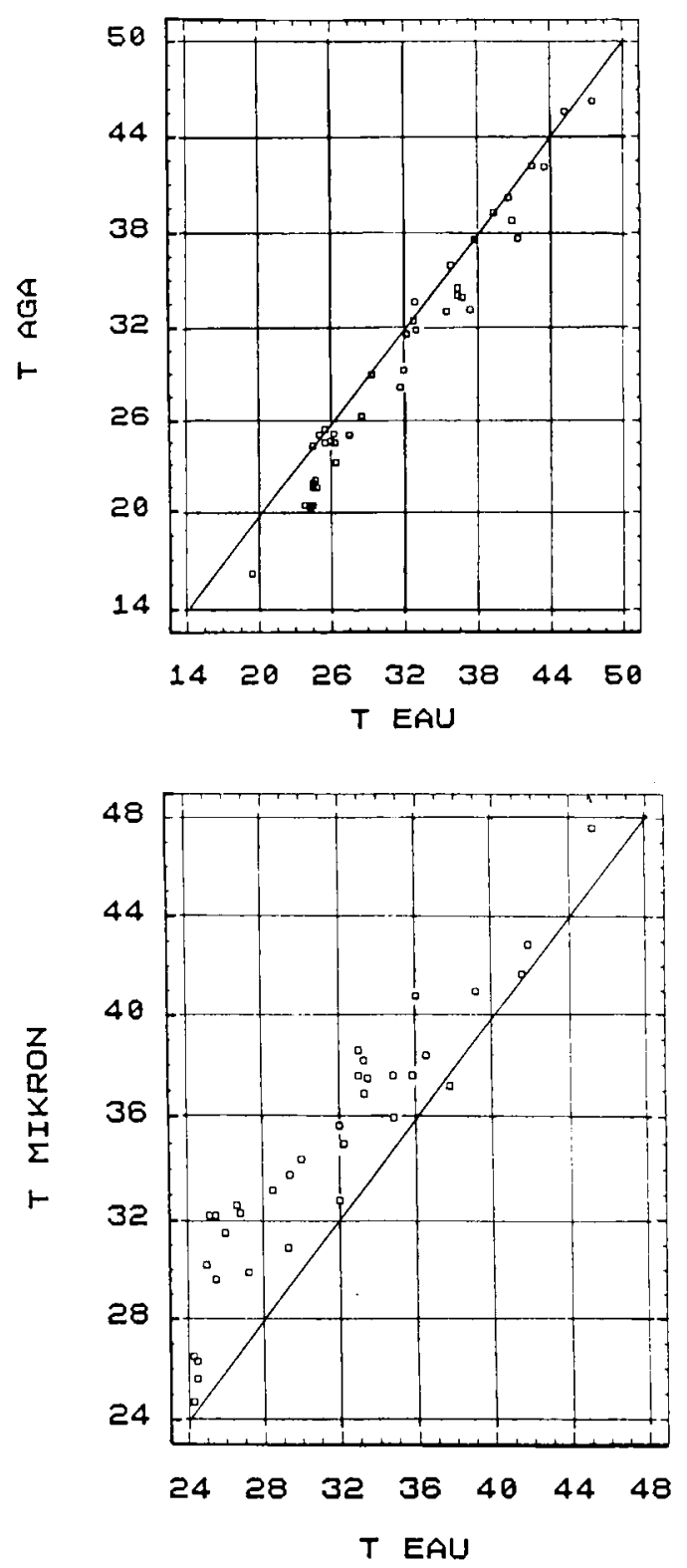

Fig 2. Relations entre les températures lues sur les radiothermometres AGA et MIKRON et la température de l'eau lors des différences journées de beau temps (mesures faites aux environs du midi solaire du 9 au 27 juillet 1990).

Les étalonnages effectués les mêmes jours sur le terrain et au laboratoire nous permettent de bien mettre en évidence l'influence des conditions de mesure. Les figures 3 et 4 montrent qu'il existe au laboratoire, comme sur le terrain, une relation linéaire étroite entre les températures indiquées et la température réelle de l'eau. Mais les pentes des droites de régression et leur ordonnée à l'origine sont différentes selon les conditions de mesure. Si $X$ est la température de l'eau et $Y$ celle indiquée par le radiothermomètre, on obtient les relations suivantes.
ETALONNGGE LABO

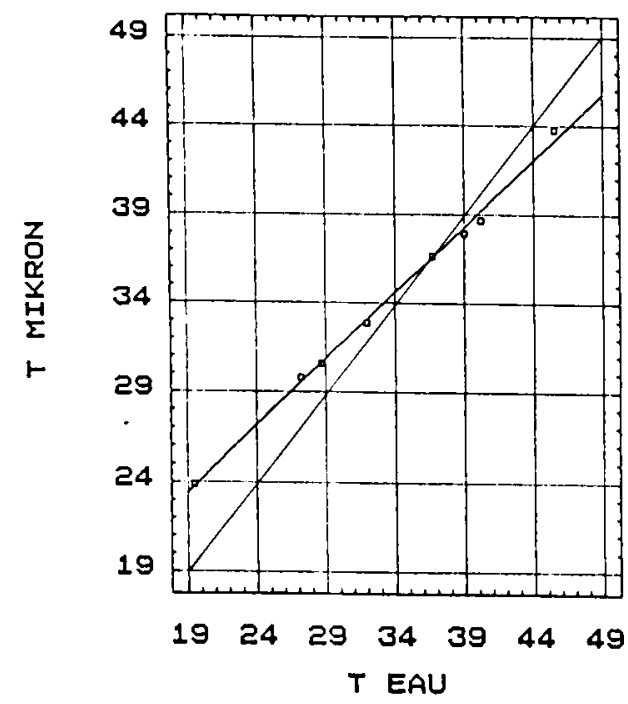

ETALONNAGE EXTERIEUR

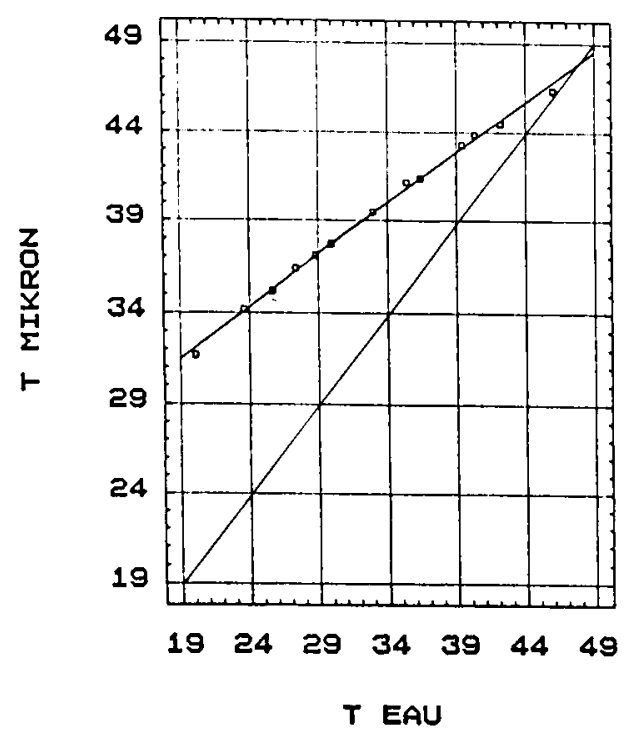

Fig 3. Relations entre les températures lues sur le radiothermomètre MIKRON et la température de l'eau lors d'étalonnages effectués au laboratoire et sur le terrain.

Pour le radiothermomètre AGA :

au laboratoire $Y=-8,50+1,11 \times R^{2}=0,998$

sur le terrain $Y=-7,50+1,17 \times R^{2}=0,994$

Pour le radiothermomètre Mikron :

au laboratoire $Y=9,26+0,74 \times R^{2}=0,997$ (3) sur le terrain $Y=20,26+0,57 \times R^{2}=0,997$ (4)

Les valeurs résiduelles des régressions linéaires sont toujours inférieures à $1,1^{\circ} \mathrm{C}$, ce qui correspond à l'ordre de grandeur de la précision des radiothermomètres. 
ETALONNAGE LABO

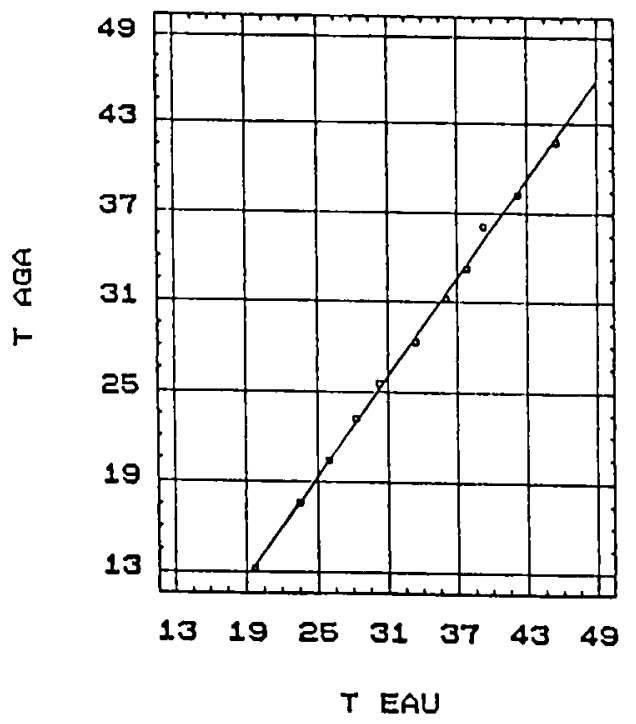

ETALONNAGE EXTERIEUR

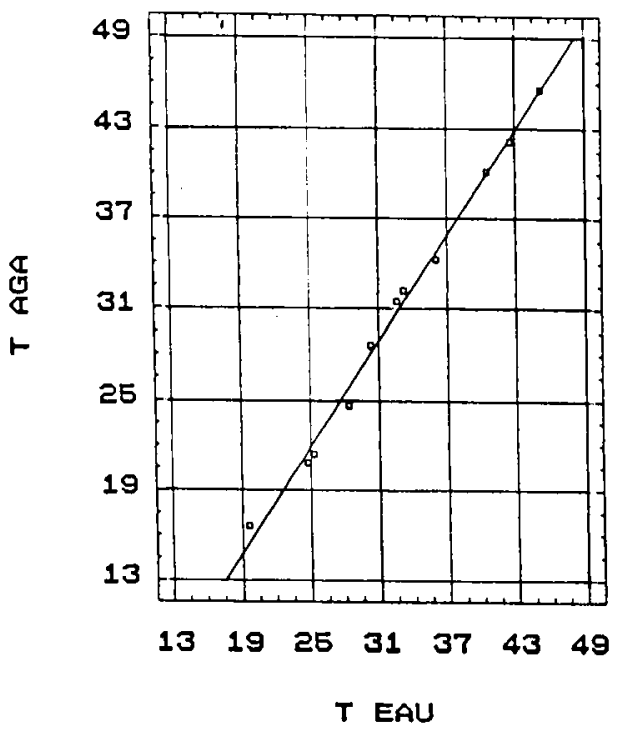

Fig 4. Relations entre les températures lues sur le radiothermomètre AGA et la température de l'eau lors d'étalonnages effectués au laboratoire et sur le terrain.

Ces résultats montrent ainsi qu'il n'est pas possible d'utiliser un radiothermomètre "pistolet" sur le terrain sans l'étalonner sur place au moment de la mesure, les résultats des étalonnages effectués au laboratoire ne pouvant pas être utilisés.

\section{DISCUSSION ET CONCLUSION}

L'analyse sommaire du fonctionnement des radiothermomètres que nous avons effectuée, a montré que les mesures de température de surface peuvent être affectées par les conditions dans lesquelles elles sont effectuées. Les résultats expérimentaux obtenus confirment les hypothèses émises. Le radiothermomètre AGA, mieux protégé contre les échauffements parasites, dérive beaucoup moins que le radiothermomètre Mikron.

La comparaison des équations (1) et (2) permet de constater que, lors des expériences présentées ci-dessus, l'effet de l'échauffement du filtre est très limité pour le radiothermomètre AGA (l'ordonnée à l'origine passe de $-8,5$ à $-7,5)$. Le changement de la pente de la droite de régression traduit un défaut de compensation de la variation de la sensibilité de la thermopile avec la température. Mais la variation reste modeste $(5 \%)$. La comparaison des équations (3) et (4) met en évidence les défauts de conception du radiothermomètre Mikron. Le détecteur, étant isolé thermiquement et recevant le rayonnement solaire même en dehors des périodes de mesure, s'échauffe fortement, ce que traduit le déplacement de l'ordonnée, à l'origine de $9,26^{\circ} \mathrm{C}$, à $20,26{ }^{\circ} \mathrm{C}$. Par ailleurs, l'échauffement du détecteur, la mauvaise prise en compte de sa température et, peut-être, l'absence de compensation de la variation de la sensibilité du détecteur avec la température, entraînent une très grande variation de la pente de la droite $(-23 \%)$. Cet appareil n'est pas recommandable pour des mesures sur le terrain.

Dans tous les cas, l'expérience limitée que nous avons effectuée devrait mettre en garde les expérimentateurs et leur montrer la nécessité d'effectuer des étalonnages sur le terrain s'ils veulent pouvoir disposer de données fiables de température radiative. II faut donc modifier les protocoles expérimentaux et prévoir de viser alternativement les cultures, et au minimum, 2 températures de référence ( 2 boîtes isothermes avec de l'eau agitée), l'une inférieure et l'autre supérieure à celle des objets visés puisque la pente et l'ordonnée à l'origine des courbes d'étalonnage varient simultanément. De plus, il sera bon de prendre en plus 1 ou 2 points intermédiaires, au cours de la série de mesures sur le terrain de manière à être sûr de la droite d'étalonnage.

\section{RÉFÉRENCES}

Amiro BD, Thurell GW, Gilles Pie TJ (1983) A small infrared thermometer for measuring leaf temperature in leaf chambers. J Exp Bot 34 148, 1569-1576 
Gaussorgues $G$ (1984) La thermographie infrarouge. Tech Doc, Paris $481 p$

Graham MED, Thurtell GW, Kidd GE (1989) Calibration of a small infrared sensor for measuring leaf temperature in the field: non steady state. Agric For Meteorol 44, 295-305

Jackson RD, Idso SB (1969) Ambiant temperature effects in infrared thermometry. Agron J 61, 324-325

Jackson RD, Robinson BF (1985) Field evaluation of the temperature stability of a multispectral radiometer. Remote Sens Environ 17, 103-108
Kustas WP, Choudhury BJ, Inoue J, Pinter PJ, Moran MS, Jackson RD, Reginato RJ (1990) Ground and aircraft infrared observations over a partially vegetated area. Int J Remote Sens 11, 3, 409-427

Lagouarde JP (1979) Mesure de la température du sol en conditions naturelles et son application en micrométéorologie. Thèse Docteur-Ingénieur, Univ Sci Médic, Grenoble

Robinson BF, Buckley RE, Burgess JA (1981) Performance evaluation and calibration of a modular multiband radiometer for remote sensing field research. Soc Photo Opt Instrum Eng 308, 146-157 